\title{
Strategy for high-precision luminosity measurement with the CMS detector at the HL-LHC
}

\author{
Francesco Romeo ${ }^{a, *}$ on behalf of the CMS Collaboration \\ ${ }^{a}$ Department of Physics and Astronomy, Vanderbilt University, Nashville, TN, 37235, USA \\ E-mail: fromeo@cern.ch
}

The High-Luminosity Large Hadron Collider (HL-LHC) upgrade of the LHC is foreseen to reach an instantaneous luminosity at a factor of five to seven times the nominal LHC design value $\left(10^{34} \mathrm{~cm}^{-2} \mathrm{~s}^{-1}\right)$. The resulting, unprecedented requirements for background monitoring and luminosity measurement create the need for new high-precision instrumentation at the Compact Muon Solenoid (CMS) experiment, using radiation-hard detectors. This contribution presents the strategy for bunch-by-bunch online luminosity measurement based on various detector technologies. The potential of the exploitation of the tracker endcap pixel detector, the outer tracker, the barrel muon detectors and the $40 \mathrm{MHz}$ muon scouting system is discussed together with the concept of a standalone luminosity and beam-induced background monitor using Si-pad sensors.

*** The European Physical Society Conference on High Energy Physics (EPS-HEP2021), ***

*** 26-30 July $2021 * * *$

*** Online conference, jointly organized by Universität Hamburg and the research center DESY ***

\footnotetext{
${ }^{*}$ Speaker
} 


\section{Introduction and motivations for high-precision luminosity measurement}

The High-Luminosity Large Hadron Collider (HL-LHC) is an upgrade of the LHC that aims to achieve instantaneous luminosities levelled to at a peak of $5 \times 10^{34} \mathrm{~cm}^{-2} \mathrm{~s}^{-1}$ (with an average number of interactions per bunch crossing, or "pileup", of $\approx 140$ ), and an ultimate performance scenario of $7.5 \times 10^{34} \mathrm{~cm}^{-2} \mathrm{~s}^{-1}$, with pileup of $\approx 200$ [1]. The timeline of the project, along with the expected peak instantaneous and integrated luminosities are illustrated in Fig. 1 (left). The unprecedented requirements of this luminosity delivery program for both background monitoring and luminosity measurement create the need for new high-precision instrumentation at the Compact Muon Solenoid (CMS) experiment, using radiation-hard detectors. In CMS, both background monitoring and luminosity measurement are under the responsibility of the Beam Radiation, Instrumentation, and Luminosity (BRIL) group.

The need for a high-precision luminosity measurement is well motivated by its impact on the high-precision studies of the LHC physics program in the HL-LHC era [2]. Figure 1 shows two important examples of precision measurements for the Higgs boson production cross sections (center) and the rapidity $\left|y^{Z}\right|$-differential $Z$ boson cross section in the dimuon final states (right) using $3000 \mathrm{fb}^{-1}$ and with the corresponding expected uncertainties. Notably, the luminosity uncertainty will dominate the experimental systematic uncertainty even with a target offline $1 \%$ precision and will remain significant even when including the expected theoretical uncertainties. The possibility to reach a $1 \%$ offline luminosity uncertainty at HL-LHC has been demonstrated with the Run2 luminosity measurements for the years 2015-16 using pp collision data at a center-of-mass energy of $13 \mathrm{TeV}$ that has achieved a precision of 1.2-1.6\% [3]. The strategy to aim toward 1\% (2\%) online (offline) luminosity measurement with the CMS detector at the HL-LHC is presented below, while a detailed description can be found in the CMS BRIL Technical Design Report (TDR) [4].
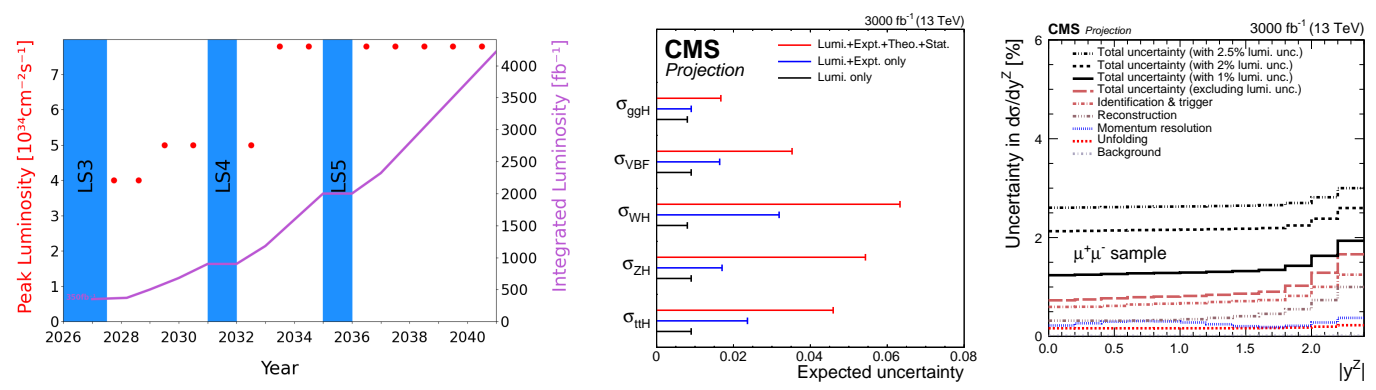

Figure 1: Left: the expected peak instantaneous (red dots) and the integrated (purple line) luminosities in the HL-LHC era [1]. Center: the expected $\pm 1 \sigma$ uncertainties in the Higgs boson production cross sections per production mode with $3000 \mathrm{fb}^{-1}$. Right: the relative systematic uncertainties from various sources in rapidity $\left|y^{Z}\right|$-differential $Z$ boson cross section measurements in the dimuon final states with $3000 \mathrm{fb}^{-1}$.

\section{Strategy overview and proposed instrumentation}

The luminosity measurements rely on the precise determination of event rates observed within the acceptance of a given luminometer. Luminosity calibration is achieved via a detector- and method-specific constant, the visible cross section $\left(\sigma_{\text {vis }}\right)$, which relates the measured event rate in the luminometer $(\mathrm{d} N / \mathrm{d} t)$ to the instantaneous luminosity $\left(\mathcal{L}_{\text {inst }}\right)$ through the relation $\mathrm{d} N / \mathrm{d} t$ $=\mathcal{L}_{\text {inst }} \sigma_{\text {vis }} . \quad \sigma_{\text {vis }}$ is measured using the van der Meer (vdM) transverse beam-separation scan 
method [5] in a low pileup regime. $\mathcal{L}_{\text {inst }}$ is measured relying on different counting methods, based on the given luminometer. Per bunch luminosity measurements will be aggregated and stored with a timing granularity of a so-called "lumi word", a unit of time expected to be equal to $\approx 1 \mathrm{~s}$ at HL-LHC, also referred to as Phase-2 for what concerns the detectors'upgrade.

The contributions to the luminosity uncertainty arise mainly from the $\sigma_{\text {vis }}$ determination together with the linearity response and stability, or efficiency, over time of the luminometer. The linearity response with respect to the instantaneous luminosity is crucial for the calibration through $\sigma_{\text {vis }}$ to be valid at high pileup. The stability response is crucial to allow a correct integration of $\mathrm{d} N / \mathrm{d} t$ over extended data-taking periods. Any ideal luminometer should exhibit perfect linearity and stability. Below, we will show the expected performance in terms of linearity as the relative difference between the mean number of observed counts and the values of a linear fit of the these counts as a funtion of the simulated pileup. Given the complexity of the measurement, its need to be carried out for each bunch crossing, and the inevitable possibility for a system to fail from time to time, in addition to effects of radiation damage over time, it is the CMS strategy to have as many independent luminometers as is practicable. Having them, their luminometer ratios for instantaneous and integrated luminosity values give a clear understanding of the overall systematics due to efficiency and nonlinearity. The CMS strategy calls for a minimum of three such fully independent systems, with ideally a total of five, that are based on diverse detector technologies and counting methods, in order to have orthogonal systematic uncertainties.

The proposed instrumentation is shown in Fig. 2 together with their operational capabilities. Three online luminometers (Disk 4 Ring 1 "D4R1", Fast Beam Condition Monitor "FBCM", and hadron forward calorimeter "HF") are expected to be almost independent of any central CMS service. They will use different technologies and services (cooled semiconductor vs. Cherenkov radiation detector), count different types of objects (clusters, hits, energy sums), as well as require external triggers or be triggerless. As a result, at least one of them shall be available $100 \%$ of the time and their uncertainties will be largely orthogonal. In the following, we will discuss the new Tracker Endcap Pixel Detector (TEPX), its D4R1, the Outer Tracker Layer 6 (OT L6), the FBCM, and the upgraded Muon Barrel (MB) and $40 \mathrm{MHz}$ muon scouting systems, while the HF [3] and the Radiation and Environment Monitoring Unified Supervision (REMUS) [3] systems are already in use and will continue operating during HL-LHC practically unchanged. The overarching philosophy behind the plans for Phase-2 is to further strengthen commonality for the BRIL systems for what concern the data acquisition, the dedicated trigger infrastructure, the readout back-end electronics, the histogramming firmware modules, and the data analysis tools.
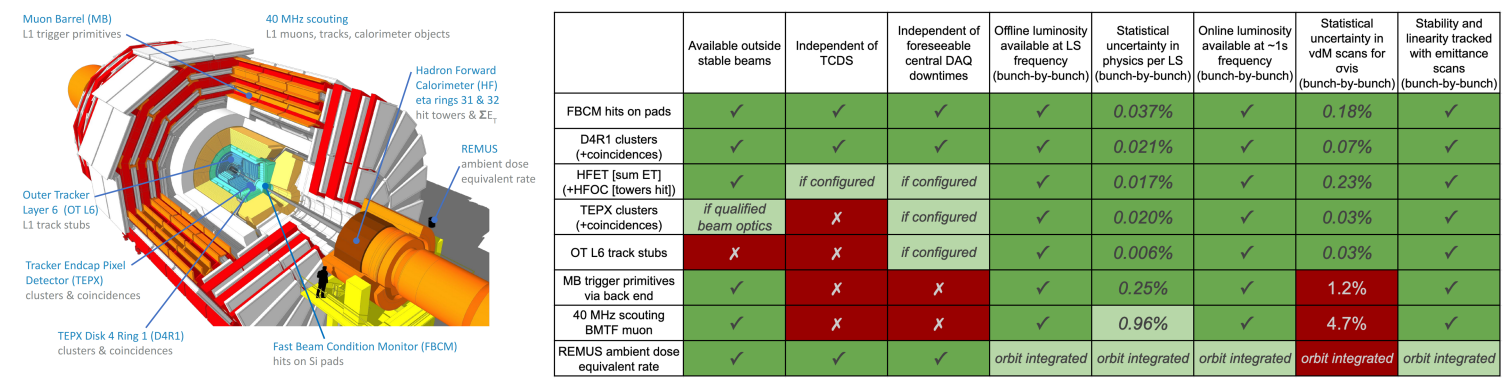

Figure 2: Subsystems for CMS Phase-2 luminosity measurement and their operational capabilities. 


\section{TEPX and D4R1 for luminosity measurement}

The TEPX [4] is located in the very forward extremity of the tracker volume, spanning a $|z|$ range from 175 to $265 \mathrm{~cm}$ and a radius between 63 and $255 \mathrm{~mm}$, as shown in Fig. 3 (left). It is composed of four large double disks per end of CMS, each made up of five individual rings with a varying number of pixel modules, for a total of more than 800 million individual pixels distributed over an area of $2 \mathrm{~m}^{2}$. It is designed to work with a maximal readout rate of 1000 $\mathrm{kHz}$ leaving some margin considering the foreseen nominal trigger accept frequency at hardware level (L1) of $750 \mathrm{kHz}$. Due to its high granularity the occupancy of the detector will be low. The luminosity measurement is performed real-time using the already established pixel cluster counting technique [3] implemented on a Field Programmable Gate Array (FPGA). The geometry of the modules and their overlap areas, as illustrated in Fig. 3 (right), allow construction of two-fold and three-fold coincidences that can be used as an additional handle on luminosity linearity and stability.

The innermost ring of the last disk in $|z|$ that lies beyond $|\eta|=4$ is the D4R1. Due to the very few tracking points at that pseudorapidity, and thus its limited use for tracking, will be operated exclusively for luminosity and beam-induced background measurements. It will be designed and cabled such that all services for D4R1 are mostly independent from the rest of TEPX and the Inner Tracker and thus can be powered and operated separately. The system can benefit from the full $825 \mathrm{kHz}$ trigger rate (while the rest of TEPX will have $750 \mathrm{kHz}$ from physics triggers and only an additional $75 \mathrm{kHz}$ of unbiased luminosity triggers). Thus, even with its smaller surface $\left(190 \mathrm{~mm}^{2}\right)$, it is expected to provide similar statistical performance as the rest of TEPX. The TEPX and D4R1 performance in terms of linearity is shown in Fig. 4.
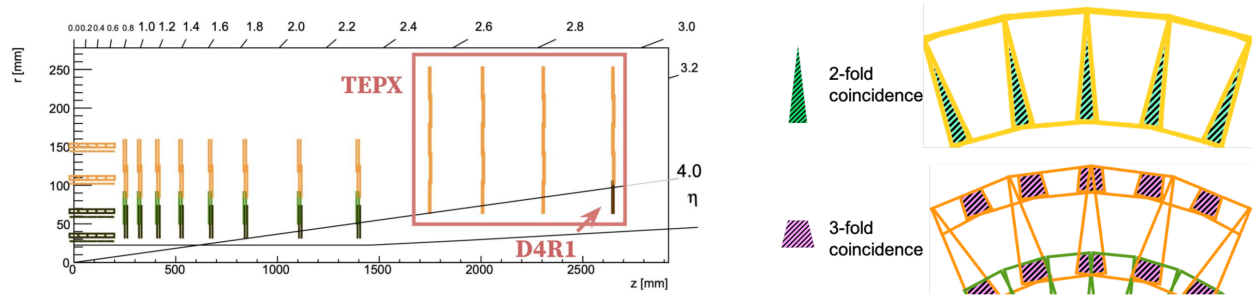

Figure 3: Left: schematic view of one quarter of the Inner Tracker in the $r-z$ plane. The four disks in the top right representing TEPX and D4R1 are highlighted. Right: drawing of the overlap areas between modules in $\phi$ and $r$, allowing the construction of two-fold and three-fold coincidences.

\section{OT for luminosity measurement}

The OT [4] consists of six barrel layers (three pixel-strip layers, TBPS, and three two-sided strip layers, TB2S) and five endcap (TEDD) disks. Layer 6 of TB2S consists of 76 ladders spanning the full azimuthal range on each end of the CMS detector, with each ladder containing 12 modules made of two Si-strip sensors, as shown in Fig. 5 (left). The OT will provide a source of high-rate physics objects - hit coincidences on the two sensors of the module, called "L1 track stubs"- for luminosity measurement. The track stub reconstruction is performed by the front-end Application Specific Integrated Circuit (ASIC). The stub information is sent to the back end at the full bunchcrossing frequency of $40 \mathrm{MHz}$. Studies using CMS Phase-2 simulations show good linearity in the counting of these objects up to a pileup of 200, as can be seen from Fig. 5 (right). 

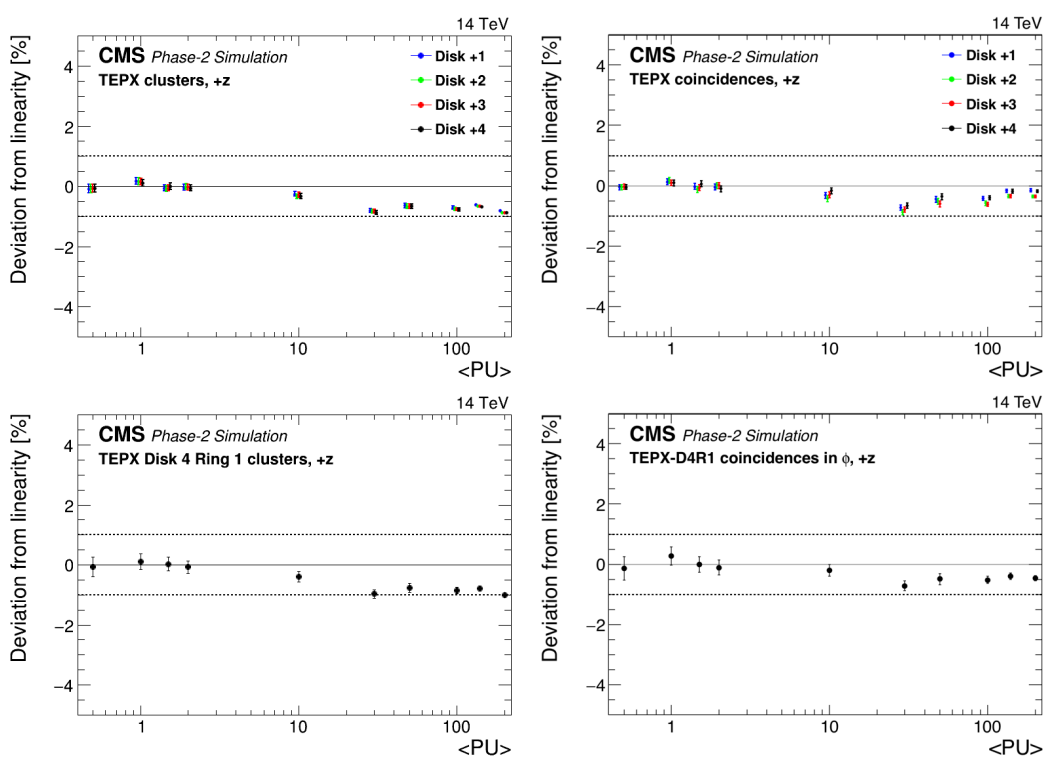

Figure 4: Deviation from linearity for clusters (left) and total coincidences (right) as a function of PU for the four TEPX disks (up) and D4R1 (down).
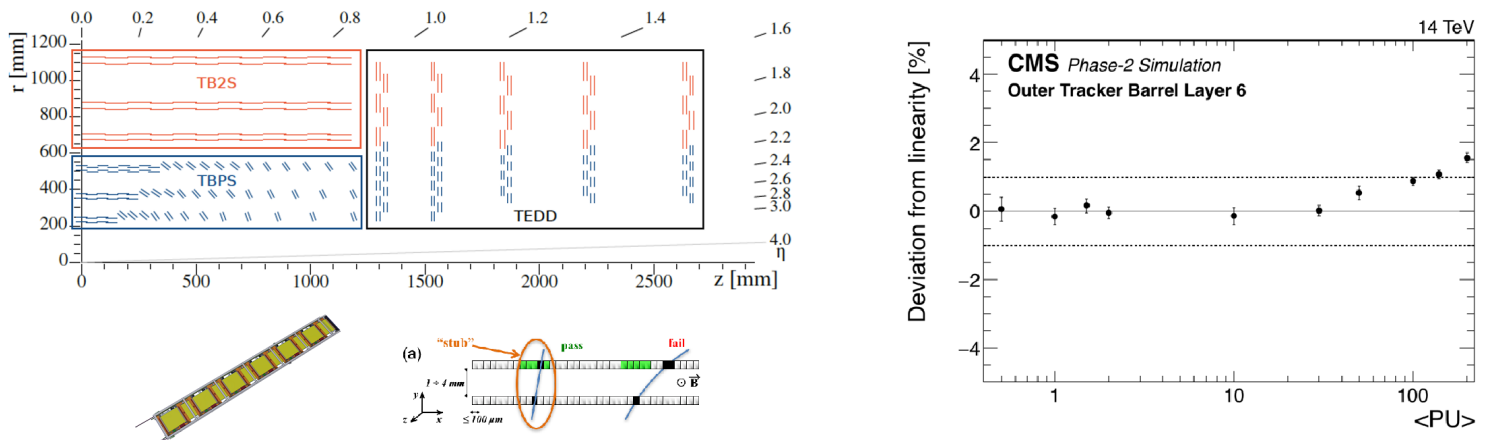

Figure 5: Left: longitudinal view of the CMS Phase-2 OT layout showing the two barrel regions (TB2S, TBPS) and the endcap region (TEDD) [4]. For the luminosity measurement, only the outermost barrel layer 6 in the TB2S part is used. This layer consists of 76 sensor ladders on each end of CMS, each ladder having 12 modules, as shown in the bottom left together with an illustration of the track stub reconstruction. Right: deviation from linearity for the mean number of stubs for barrel layer 6 .

\section{FBCM for luminosity and beam-induced background}

The FBCM is a proposed standalone luminometer designed to run independently from the CMS data taking and with mostly orthogonal systematics compared to those of the other luminometers. It is composed of 336 silicon-pad sensors, each with a currently chosen area of $2.89 \mathrm{~mm}^{2}$ and a thickness of $300 \mu \mathrm{m}$, with fast front-end chips wire-bonded adjacent to the sensors. It is organized in 4 quarters with the proposal to install it behind disk 4 of the TEPX system, close to the bulkhead, corresponding to $8<r<30 \mathrm{~cm}$ and $277<|\mathrm{z}|<290 \mathrm{~cm}$, as illustrated in Fig. 6 (left and center). At the back end, an Advanced Telecommunications Computing Architecture (ATCA) board performs the digital processing required for the zero-counting algorithm [3] to measure the luminosity and 
beam-induced background. Good performance is expected for the luminosity measurement as reported in Fig. 6 (right), which shows the expected deviation from linearity versus pileup.

Beam-induced background measurement is another potential strength of FBCM because of its capability of fast sub-bunch crossing measurement that can distinguish between the products of nominal collisions and the incoming beam-induced particles within two separate bunches. In order to be identified against more overwhelming out-of-time background contributions, beam-induced background is measured from a bunch crossing where there is a sufficiently long time interval without collisions (a bunch crossing preceded by at least $\approx 30$ empty bunch crossings), such as the beginning of a bunch crossing in a series of bunches, or noncolliding bunches.
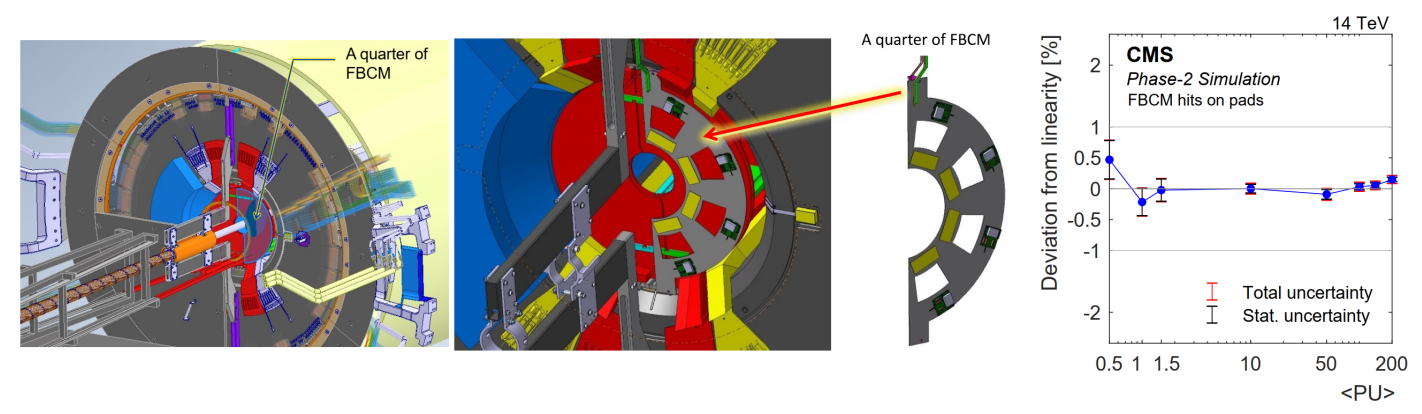

Figure 6: Left: sketch of a location under consideration for FBCM behind the last disk of TEPX. Center: a 3D model of a potential mechanical concept for an FBCM quarter, viewed from the side away from the IP. Right: the expected deviation from linearity versus pileup.

\section{MB and $40 \mathrm{MHz}$ muon scouting system for luminosity measurement}

The counting of muon track stubs at L1 trigger level using the muon barrel detector, including Drift Tubes (DT) chambers and the Resistive Plate Chambers (RPCs), has proven to be useful for the luminosity measurement because of its excellent linearity and stability [3]. For this reason, it is considered also for HL-LHC luminometry despite the method's smaller statistical power. The counting will be carried out with the use of dedicated BRIL firmware and improved with respect to Run 2 by upgrading the system from measuring orbit-integrated to bunch-by-bunch rates.

Muon candidate counting at L1 trigger level will also be exploited relying on the information of the entire muon detector (barrel and endcap) using a different technique known as the $40 \mathrm{MHz}$ scouting [6]. The $40 \mathrm{MHz}$ muon scouting system receives data from the L1 trigger over spare output links and is able to process them in real time and to run largely independently of the standard CMS global trigger, with the advantage of not suffering from rate limitation and biases associated with the L1 trigger combination algorithms. A demonstrator of the scouting system was already tested at CMS and its use will be extended during the coming data-taking before HL-LHC.

The performance of these muon-based luminometers are shown in Fig. 7 that illustrates the deviation from linearity for the total MB trigger primitive rate from the 3 DT stations (MB1, MB2, and MB3) (left) and the barrel muon track finder (BMTF) counting rate from the $40 \mathrm{MHz}$ scouting system (right) as a function of the instantaneous luminosity measured by the HF detector during part of the 2018 data-taking. 

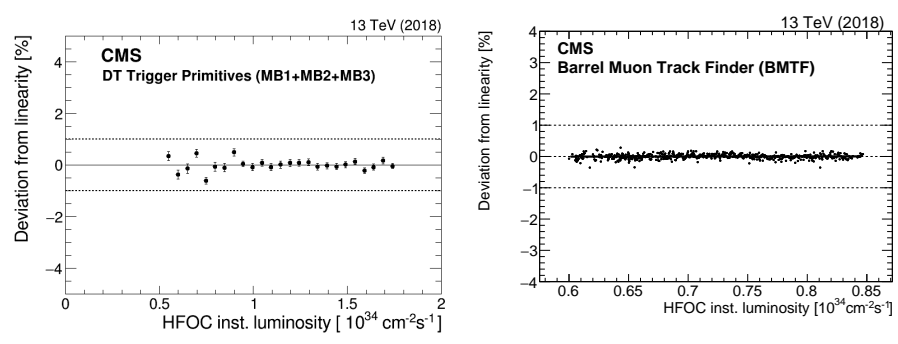

Figure 7: Deviation from linearity of the total MB trigger primitive rate from the 3 DT stations MB1, MB2, and MB3 (left) and the barrel muon track finder counting rate from the $40 \mathrm{MHz}$ scouting system (right) as a function of the instantaneous luminosity as measured by the HF detector during part of the 2018 data-taking.

\section{Summary}

The HL-LHC era presents new challenges for luminosity and beam-induced background measurements because of the increased instantaneous luminosity of a factor of five to seven times the nominal LHC design value $\left(10^{34} \mathrm{~cm}^{-2} \mathrm{~s}^{-1}\right)$ with a pileup up to $\approx 200$ [1], and a total integrated luminosity up to $4000 \mathrm{fb}^{-1}$. The need for a high-precision luminosity measurement is motivated by the demands of foreseen physics measurements. CMS has a well-defined upgrade plan described in detail in the CMS BRIL TDR [4] that has been approved by the LHC Experiments Committee (LHCC). The CMS BRIL group intends to fulfill this task by relying on different approaches exploiting a diverse set of Phase-2 CMS detector systems (TEPX, BRIL-operated D4R1, and OT L6); a new dedicated luminometer (FBCM); and the upgrade of already established or demonstrated technology (MB, $40 \mathrm{MHz}$ muon scouting, HF, and RAMSES systems). All of the luminometers are designed to exhibit excellent linearity and stability to achieve the targeted $1 \%(2 \%)$ online (offline) precision.

\section{References}

[1] I. Béjar Alonso, O. Brüning, P. Fessia, L. Rossi, L. Tavian, and M. Zerlauth, "High-Luminosity Large Hadron Collider (HL-LHC): Technical design report," CERN, 2020.

[2] ATLAS and CMS Collaborations, "Addendum to the report on the physics at the HL-LHC, and perspectives for the HE-LHC: Collection of notes from ATLAS and CMS," CERN, 2019.

[3] A. M. Sirunyan et al., "Precision luminosity measurement in proton-proton collisions at $\sqrt{s}=$ $13 \mathrm{TeV}$ in 2015 and 2016 at CMS," Eur. Phys. J. C, vol. 81, p. 800, 2021.

[4] CMS Collaboration, "The Phase-2 upgrade of the CMS tracker," Technical Design Report CERN-LHCC-2017-009, CMS-TDR-014, 2017. [Online]. Available: https: //cds.cern.ch/record/2272264

[5] S. van der Meer, "Calibration of the effective beam height in the ISR," Tech. Rep. CERN-ISR-PO-68-31, 1968. [Online]. Available: https://cds.cern.ch/record/296752

[6] G. Badaro et al., "40 MHz level-1 trigger scouting for CMS," EPJ Web Conf., vol. 245, p. 01032, 2020. 